\title{
Design and Develop an Information system for Court Data in the Republic of Iraq by using SSRS Reports with SSAS Cubes
}

\author{
Ayad Mohammed Jabbar \\ Computer Science Department \\ Shatt Al-Arab University College \\ Basra, Iraq \\ ayadjb@gmail.com
}

\begin{abstract}
Multidimensional Online analytical processing (MOLAP) technology is considered a good tool to produce meaningful and quality results by using a multidimensional cube. The term "multidimensional cube" is used to refer to the multiple layers of data that are used to show the result. This result is identified by high-level management to increase the Iraqi court work and to improve its quality. The Iraqi court needs an analytical report to make a strategic decision on case date, case type, case state, judge, criminal age, and criminal gender. Currently, MOLAP is known as the best and strongest technique because it provides rapid, dynamic, and multiple analyses of data; presents knowledge from different perspectives; comes up with data in time series intervals; and drills down into multiple levels of data layers to present different types of details. The SQL Server Reporting Service (SSRS) presents analysis reports based on the MOLAP cube. This paper focuses on designing and developing the analysis reports of the court data system of the Republic of Iraq by using SSRS with SQL Server Analysis Service to create the MOLAP cubes.
\end{abstract}

Index Terms - Court Data, SSRS Reports, SSAS Cubes, SSIS, MOLAP, ETL, DW, HTTP.

\section{INTRODUCTION}

The criminal court of Iraq is based on an inquisitorial system and consists of two chambers: an investigative court and a criminal court. The court was created by the Coalition Provisional Authority in 2003 to handle cases involving serious crimes, such as government corruption, terrorism, and organized crime, that were previously handled by governorate level judges in the ordinary criminal courts [1]. Database systems are deployed in majority of the courts to save and retrieve data. However, the high level management needs a good strategic plan and decision making tools to achieve their mission with minimum effort and time [2].

The decision maker needs the data to be presented in a manner that is easy to understand, such as tables and charts [3]. The court does not have an information system for monitoring, analyzing, and managing the criminal cases in Iraq. Increasingly large amounts of judicial data have been stored, and the volume is still increasing. However, despite this wealth of data, the judicial system has been unable to capitalize on its value fully because information that is implicit in the data is difficult to discern [4]. The problems with the current database are as follows: information is too detailed for analysis, different locations, different coding, and different design techniques. SSRS is a technique to create SQL Server Reporting Services reports by using SQL Server Analysis Service (SSAS) cubes as data sources among relational data, which have an intuitive interface and take full benefit of SSAS performance optimization features, is explained in this paper $[5,6]$.

\section{Prototype DeVelopment AND ChALLENGES}

All data, definitions, and indices are stored in an multi SQL database management system. Our proposed system involves directly extracting useful court data from a relational database. The information is needed to aid government studies. 
The list of requirements for the reports is based on frequently asked questions as shown in Table I.

TABLE I

\begin{tabular}{|c|l|l|}
\multicolumn{4}{|c}{ ANALYTICAL REPORT REQUIREMENTS } \\
\hline No. & \multicolumn{1}{|c|}{ Requirements } & Priority \\
\hline 1 & $\begin{array}{l}\text { The system user needs to } \\
\text { determine the number of judges } \\
\text { by state, case, and time. }\end{array}$ & High \\
\hline 2 & $\begin{array}{l}\text { The system user needs to } \\
\text { determine the number of judges } \\
\text { by gender, age, cases, and time. }\end{array}$ & High \\
\hline 3 & $\begin{array}{l}\text { The system user needs to } \\
\text { determine the number of judges } \\
\text { appearing for each gender by } \\
\text { time. }\end{array}$ & Medium \\
\hline 4 & $\begin{array}{l}\text { The system user needs to } \\
\text { determine the number of judges } \\
\text { for each age by time. }\end{array}$ & Medium \\
\hline 5 & $\begin{array}{l}\text { The system user needs to } \\
\text { determine the number of judges } \\
\text { for each case by time. }\end{array}$ & High \\
\hline 6 & $\begin{array}{l}\text { The system user needs to } \\
\text { determine the number of judges } \\
\text { appearing for each state by time. }\end{array}$ & High \\
\hline
\end{tabular}

These requirements were determined using only the research obtained from previous research papers and not from an actual hands-on evaluation tool. For this reason, a different set of opinions will be available once the tool is implemented. This step is important because future decision-making will be based on past data, which are stored in the current system. The main purpose of the research is to establish the feasibility of analysis in the court system and to determine how to optimally share this technology to non-technical users. This paper aims to achieve the following objectives:

- To understand low-level data to create helpful analytical reports from them.

- To determine the online analytical processing (MOLAP) technique to be used in our system.

- To design and develop an interface for the analytical reports.

Relational data are the original sources of data. Relational data control and run fundamental business tasks. They are highly normalized with many tables. However, the information we need to obtain is needed for planning, problem solving, and decision support. Data have multidimensional views of various types of activities. These data are typically de-normalized with fewer tables. However, data stores are in different locations; data coding is inconsistent; and different data representations exist, as shown in Table II. In this research, we use star schema data warehouse (DW) to obtain the information needed.

TABLE II

DIFFERENCE OF DATA REPRESENTATIONS.

\begin{tabular}{|c|c|c|}
\hline Data Base 1 & Data Base 2 & Data Base 3 \\
\hline Male & $\mathrm{M}$ & 0 \\
\hline Female & $\mathrm{F}$ & 1 \\
\hline
\end{tabular}

The majority of development work for MOLAP projects comprises design and implementation of Extract, Transfer and loading (ETL) activities. However, our data are distributed across various databases, systems, and places and are stored in various media types. We want to convert our data into useful information. The ETL process is defined as the development and compilation of programs or script, which extracts data from database sources, transforms them, and loads them in DWs based on the user's requirements [7].

A good ETL process design is maintainable, flexible, and efficient. Researchers divided the ETL process into five modules, namely, data extraction, data validation, data cleaning, conversion, and loading to the target destination [8], as shown in Fig.(1).

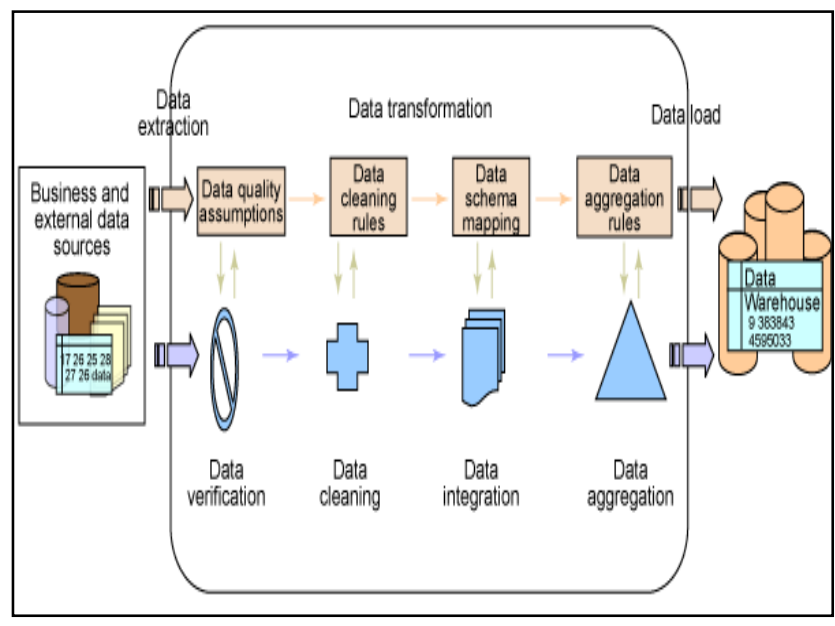

Fig.1 ETL Process. 
We have to create our DW before we start to prepare the MOLAP cube. The DW is the database specifically designed to facilitate analysis and decision-making activities. DW is integrated, non-volatile, subject-oriented, and time-variant data storage. Creating a DW helps convert data into helpful information; DW provides multiple dimensions through which the data are studied. Thus, high-level management can make fast decisions using the data. The data can also be utilized for data mining and informative dashboards in the future. The collection of operational data from various sources and applications can be conducted using ETL. This approach involves three steps: extraction of data from heterogeneous sources, transformation process, and loading of data into DWs [9]. DW is used in our system is star schema. Star schema architecture is the simplest warehouse design. The main features of the star schema are a table at the center, called the "fact table," and the dimension tables that allow browsing of specific categories, summarizing, drill-downs, and specifying criteria [10].

We warehousing the data from different sources by using SQL Server Integration Services (SSIS). The DW is store in Microsoft SQL relational database management system. Fig.(2) shows the fact and dimension tables in the star schema for the Iraqi court system, which includes six dimension tables and one fact table. Dimension tables are tables of a dimensional model that have surrogate key columns, which are created with unique identifiers for the dimension records. The dimension table is a single-part primary key consisting of descriptive attribute columns [11]. The tables are age, classification, state, gender, case, and time. The tables have been connected to the center, which is represented by the fact table. The single table represents a transaction or event that occurs in a single period, whereas the fact table represents the single branch in the hierarchy [12]. As large number of reports requirement and due to limitations of relational database, MOLAP was appeared to provide court report with deeper understanding and knowledge about many aspects about the court as show in Fig.(3).

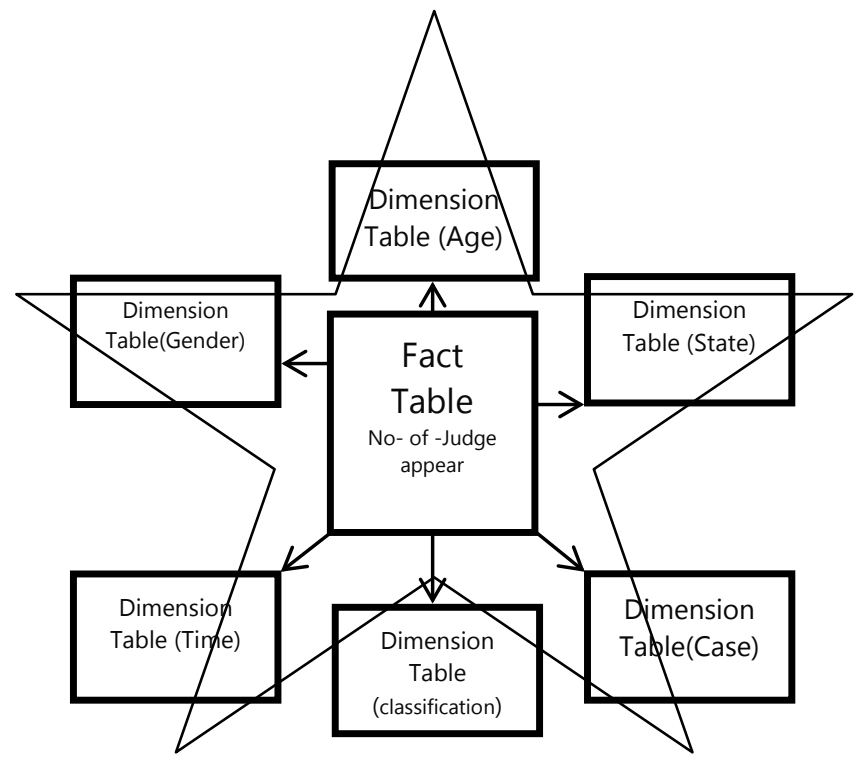

Fig.2 DW Star Schema Architecture

MOLAP, a multidimensional data cube, has gained popularity as a method to support decisions in situations where raw data with measures, such as cases or number of judges, need to be analyzed at different levels of statistical reports. In MOLAP, queries are made against a multidimensional database called an MOLAP cube, in which the dimension determines the measured values.

The dimensions of our system are time, states, age, gender, classification, and cases. The measures can be cases and number of judges. A dimension can adopt a hierarchical structure to enable the analysis to be performed daily or monthly. The MOLAP databases are aggregated from other data operations used by ETL and frequently uploaded to the DW. The current cube is implemented using SSAS which has MDX functions available to create highly complex calculations.

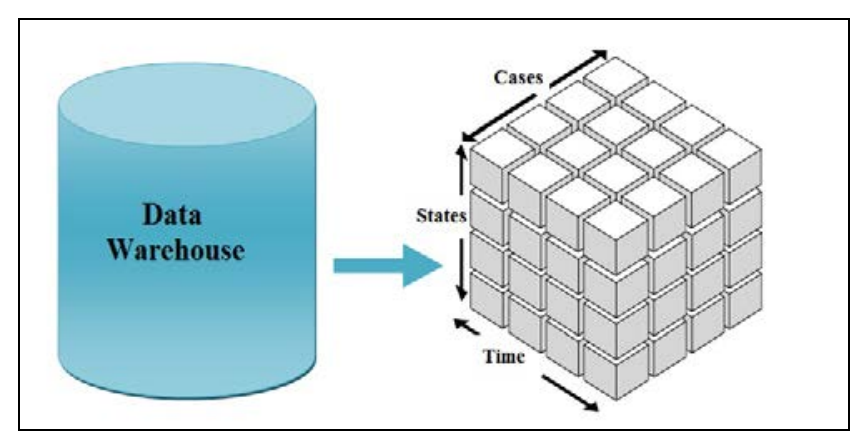

Fig.3 Multidimensional Data Cube. 
SSRS is used to create analytical reports in our system for the presentation layer. Some of the reasons we use SSRS are the following: the report development environment is a standard Microsoft platform; SSRS reports are primarily accessed via Hypertext Transfer Protocol (HTTP) or HTTP Secure; SSRS reports can be rendered to PDF, Excel, Word, and TIFF; SSRS reports can be created as SharePoint web parts; and SSRS reports can be created as a self-service alert.

SSRS gives a full range of ready-to-use tools and services to help in management, deployment, and creation of reports for our organization. The SSRS can create interactive, tabular, graphical, or free-form reports from relational, multidimensional, or XML-based data sources. SSRS can include rich data visualization, including charts, maps, and spark lines.

The system is internet-based application developed by using C\# language and includes all SSRS reports as share point web parts. The main users of our prototype are the Ministry of Justice authority officers. It enables users to analyze court data in the Republic of Iraq with high quality results and less mistakes. It also provides customer service at any time or place. This system is the first in the Iraqi court that can serve as a guide for others to develop their own application. The usage is restricted to several officers who have access rights to the data. Therefore, the system reports are equipped with system login protection. Fig.(4) displays the main web based interface of our system.

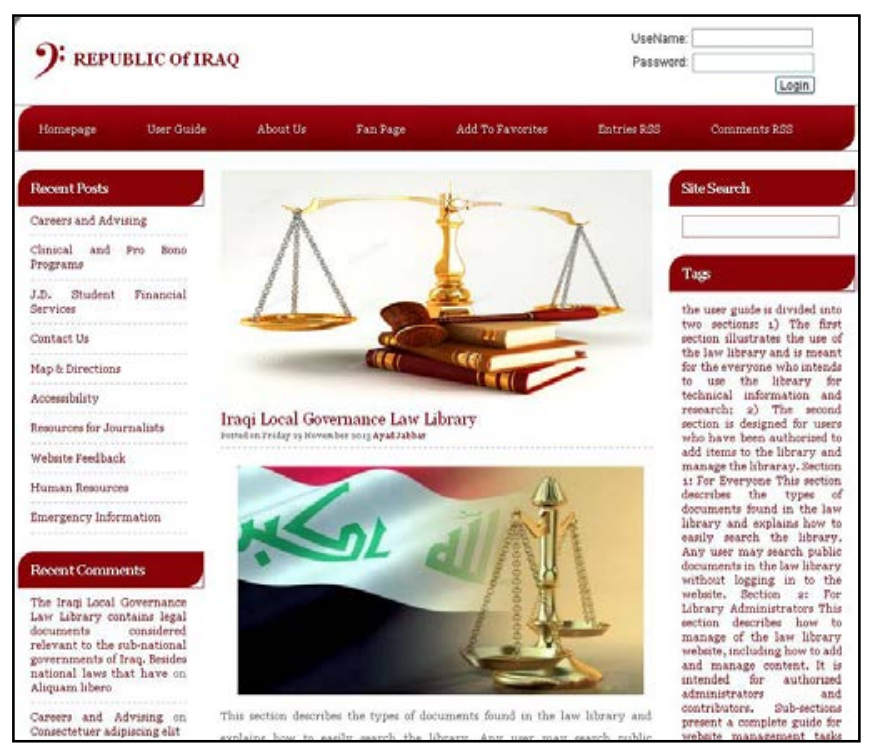

Fig.4 Main web based interface.
Once the users are logged in to the system, users could view a list of court reports. The report parameters are very flexible, which meet all the prototype requirements. Fig.(5.1) and Fig.(5.2) show some of the system reports.

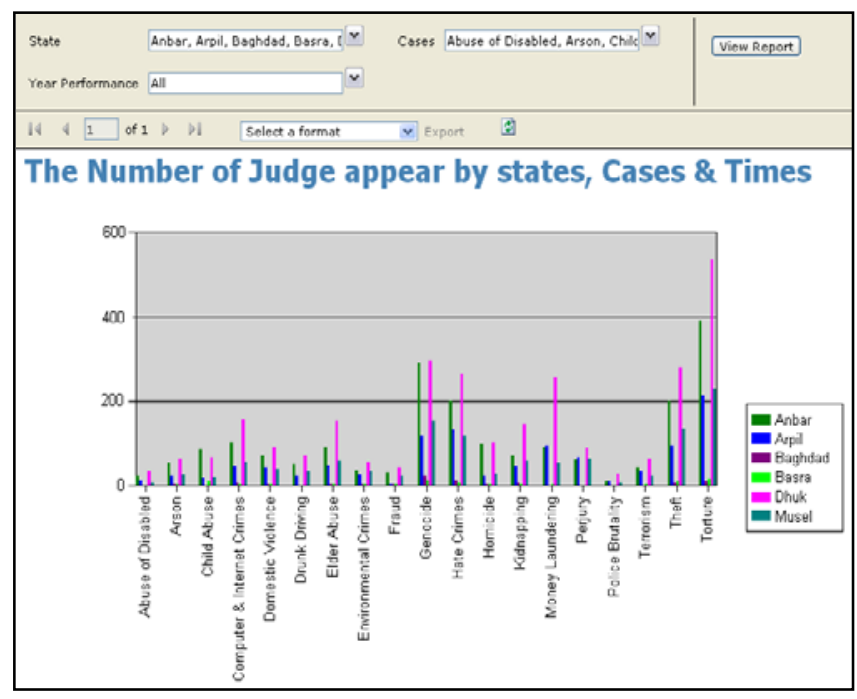

Fig.5.1 Reports of Court Data.

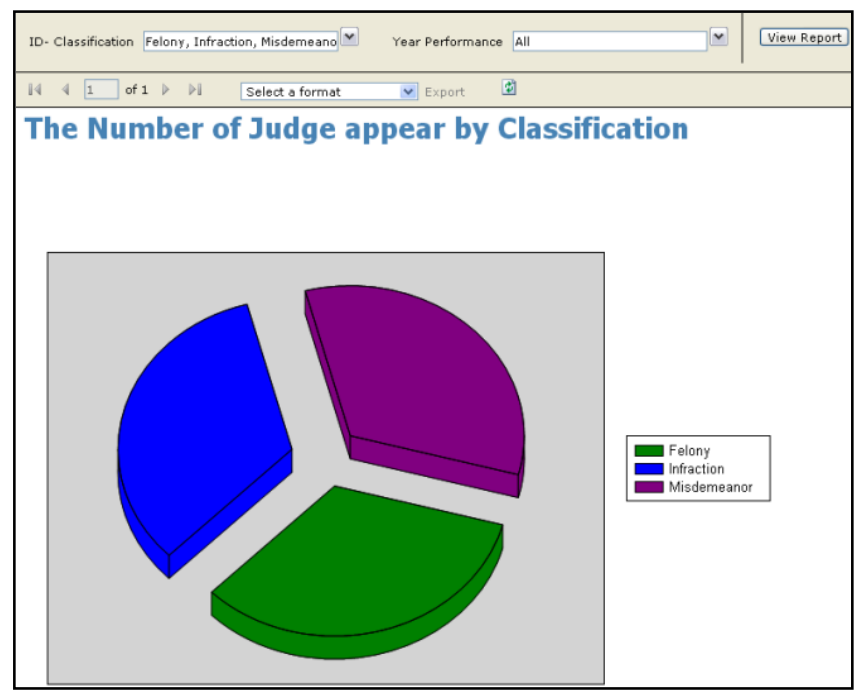

Fig.5.2 Reports of Court Data.

\section{CONCLUSION AND FUTURE WORK}

The software that has been developed for the analysis of the Republic of Iraq court data will increase knowledge of the court data, thereby allowing the authority to make good and timely decisions. Our system supports most of the functions performed in conventional operations. It also utilizes database utilities with SSRS graphic user interface, which can assist in report 
generation. These database utilities can help decision-makers forecast future impacts and plans for different crimes in different classifications, the locations of each crime, and the crime rates based on age and gender in different years.

We will use the data mining in ETL in future works to focus on data quality, which is one of the most important concerns in BI projects. By using data mining query transformation, we can allow users to modify or redirect records depending on the result of data mining prediction. In addition, we will focus on analysis service. Moreover, we plan to build BI dashboards, which can represent the key performance indicators using multidimensional analysis in real-time based strategic analysis.

\section{REFERENCES}

[1] J. Gerald M. Muhl, "DEFEATING IMPROVISED EXPLOSIVE DEVICES (IED): ASYMMETRIC THREATS AND CAPABILITY GAPS," Master, Department of Defense, The U.S. Army War College Philadelphia, 2011.

[2] E. Turban, R. Sharda, D. Delen, and T. Efraim, Decision support and business intelligence systems: Pearson Education India, 2007.

[3] A. Mohammed and K.-m. Kue Ruhana, "Graphical Web based tool for Generating Query from star Schema " in Proceedings of the 2rd International Conference on Computing and Informatics, ICOCI09, Kuala Lumpur, Malaysia,2009.

[4] O. Kufandirimbwa and C. Kuranga, "Towards Judicial Data Mining: Arguing for Adoption in the Judicial System," Online Journal of Physical and Environmental Science Research, vol. 1, pp. 15-21,2012.

[5] S. Harinath and S. R. Quinn, Professional SQL server analysis services 2005 with MDX: John Wiley \& Sons, 2006.
[6] R. Jacobsen, S. Misner, and H. Consulting, MicrosoftÂ® SQL ServerTM 2005 Analysis Services Step by Step: O'Reilly, 2009.

[7] J. Li and B. Xu, "ETL tool research and implementation based on drilling data warehouse," in FSKD '10: Seventh International Conference on Fuzzy Systems and Knowledge Discovery, Yantai, Shandong,China:IEEE, 2010, pp. 2567-2569.

[8] J. Tang, K. Cui, Y. Feng, and G. Tong, "The Research \& Application of ETL Tool in Business Intelligence Project," in IFITA '09: International Forum on Information Technology and Applications, Chengdu,China:IEEE, 2009, pp. 620-623.

[9] M. U. Shaikh, S. U. R. Malik, M. A. Qureshi, and S. Yaqoob, "Intelligent Decision Making Based on Data Mining Using Differential Evolution Algorithms and Framework for ETL Workflow Management," in ICCEA '10: Second International Conference on Computer Engineering and Applications, Bali Island,Indonesia:IEEE, 2010, pp. 22-26.

[10] C. Surajit and D. Umeshwar, "An overview of data warehousing and MOLAP technology," SIGMOD Rec., vol. 26, pp. 65-74, 1997.

[11] Z. Hua-long, "Application of MOLAP to the analysis of the curriculum chosen by students," in ASID '08: 2nd International Conference on Anti-counterfeiting, Security and Identification, Guiyang,China:IEEE, 2008, pp. 97-100.

[12] E. Malinowski and E. Zimányi, "Hierarchies in a multidimensional model: From conceptual modeling to logical representation," Data \& Knowledge Engineering, vol. 59, pp. 348-377, 2006. 